\title{
NOTE
}

\section{TOTAL RADIANCES AND EQUIVALENT WIDTHS OF DOPPLER LINES FOR NONISOTHERMAL PATHS*}

\author{
H. Y. YamaDA \\ Willow Run Laboratories, Institute of Science and Technology, The University of Michigan, \\ Ann Arbor, Michigan 48107
}

(Received 23 February 1967)

\begin{abstract}
An analytical method similar to that used by Simmons ${ }^{(1)}$ on Lorentz lines was applied to the problem of the growth of an isolated Doppler line for the case of nonisothermal paths. The results, given in terms of the total radiance $(N)$ and equivalent width $(W)$ of the line, are :
\end{abstract}

$$
\begin{aligned}
& N=(\pi / \ln 2)^{1 / 2} \gamma_{e} \int_{0}^{g\left(x_{L}\right)} N_{v}^{*}(g) d g \\
& W=(\pi / \ln 2)^{1 / 2} \gamma_{e} g\left(x_{L}\right)
\end{aligned}
$$

where

$$
g=g(x)=\sum_{n=0}^{\infty} \frac{(-1)^{n} x^{n+1}}{(n+1) !(n+1)^{1 / 2}}
$$

is the function obtained by LADENBURG ${ }^{(4)}$ for the isothermal case, $\gamma_{e}$ is an effective spectral line half-width, $N_{v}^{*}(g)$ is the Planck function of the local temperature in the emitting-absorbing gas, the nondimensional optical depth is defined by $\mathrm{d} x=(\ln 2 / \pi)^{1 / 2}[S(X) G(X) \mathrm{d} X] / \gamma_{e}$, where $S(X)$ is the local value of the line strength, $d X$ is the increment of standard optical depth, and $G(X)$ is a function characterizing the temperature dependence of the line width, and the subscript $L$ refers to the total path in the emitting-absorbing gas.

RECENTLY relations for total radiances $(N)$ and equivalent widths $(W)$ of isolated Lorentz lines along nonisothermal paths were developed using an analytical method. ${ }^{(1)}$ This note describes a similar analysis which was carried out for isolated Doppler lines.

The spectral absorption coefficient at frequency $v$ and temperature $T, k(v, T)$, in this case is

$$
k(v, T)=\left(\frac{\ln 2}{\pi}\right)^{1 / 2} \frac{S(T)}{\gamma(T)} \exp \left\{-\left[\frac{v-v_{0}}{\gamma(T)}\right]^{2} \ln 2\right\}
$$

where $S(T)$ is the line strength, $\gamma=\left(v_{0} / c\right)(2 k T \ln 2 / m)^{1 / 2}$ is the Doppler line half-width at half-maximum line intensity, $v_{0}$ is the line center, $k$ is the Boltzmann constant, and $m$ is the mass of the emitter.

* This work was supported by the Advanced Research Projects Agency, Dept. of Defense, Washington, D.C., under Contract DAHC15-67-C-0062. 
Relations for the total line radiance, $N$, and equivalent width, $W$, of an isolated Doppler line in an isothermal region are well known:(2)

$$
\begin{aligned}
N & =\left(\frac{\pi}{\ln 2}\right)^{1 / 2} \gamma N_{v}^{*} g(x) \\
W & =\left(\frac{\pi}{\ln 2}\right)^{1 / 2} \gamma g(x)
\end{aligned}
$$

where

$$
\begin{gathered}
g(x)=x\left(1-\frac{x}{2 !(2)^{1 / 2}}+\frac{x^{2}}{3 !(3)^{1 / 2}}-\frac{x^{3}}{4 !(4)^{1 / 2}}+\ldots\right) \\
=\sum_{n=0}^{\infty} \frac{(-1)^{n}}{(n+1) !} \frac{x^{n+1}}{(n+1)^{1 / 2}} \\
x=\left(\frac{\ln 2}{\pi}\right)^{1 / 2} \frac{S X}{\gamma}
\end{gathered}
$$

and $N_{v}^{*}$ is the Planck function, assumed to be constant over the spectral interval occupied by the line. For small $x, g(x) \rightarrow x$. So equations (2) and (3) have the following form for weak lines:

$$
\begin{aligned}
& N=\left(\frac{\pi}{\ln 2}\right)^{1 / 2} \gamma N_{v}^{*} x=N_{v}^{*} S X \\
& W=\left(\frac{\pi}{\ln 2}\right)^{1 / 2} \gamma x=S X .
\end{aligned}
$$

For large values of $x$, we use an asymptotic form ${ }^{(3)}$ of $g(x)$ :

$$
g(x) \cong \frac{2}{(\pi)^{1 / 2}}[\ln (x)]^{1 / 2}
$$

$N$ and $W$ in the strong line limit then are:

$$
\begin{aligned}
& N=\frac{2 \gamma}{(\ln 2)^{1 / 2}} N_{v}^{*}[\ln (x)]^{1 / 2}=\frac{2 \gamma}{(\ln 2)^{1 / 2}} N_{v}^{*}\left\{\ln \left[\left(\frac{\ln 2}{\pi}\right)^{1 / 2} \frac{S X}{\gamma}\right]\right\}^{1 / 2} \\
& W=\frac{2 \gamma}{(\ln 2)^{1 / 2}}[\ln (x)]^{1 / 2}=\frac{2 \gamma}{(\ln 2)^{1 / 2}}\left\{\ln \left[\left(\frac{\ln 2}{\pi}\right)^{1 / 2} \frac{S X}{\gamma}\right]\right\}^{1 / 2} .
\end{aligned}
$$

Tabulations of $g(x)$ for $x=0 \cdot 10$ to 1000 can be found in Ref. 4 .

In a nonisothermal region, the absorption coefficient is a function of temperature and wavelength, hence of path and wavelength since temperature can be represented as a function of conditions along the path. The equations for line radiance and equivalent width 
for nonisothermal paths are obtained from the equation of transfer:

$$
\begin{aligned}
& N=\int_{0}^{\infty} N_{v} \mathrm{~d} v=\int_{0}^{\infty} \int_{0}^{X_{L}} k(v, X) N_{v}^{*}(X) \exp \left[-\int_{0}^{X} k\left(v, X^{\prime}\right) \mathrm{d} X^{\prime}\right] \mathrm{d} X \mathrm{~d} v \\
& W=\int_{0}^{\infty}\left\{1-\exp \left[-\int_{0}^{x_{L}} k(v, X) \mathrm{d} X\right]\right\} \mathrm{d} v .
\end{aligned}
$$

If we assume $N_{v}^{*}=N_{v_{0}}^{*}$, change the integration over $v$ to an integration over $\Delta v=v-v_{0}$ and assume no contribution from $-\infty$ to $-v_{0}$ so the limits of integration change from 0 to $+\infty$ to $-\infty$ to $+\infty$, equations (11) and (12) then become:

$$
\begin{aligned}
& N=\int_{-\infty}^{\infty} \int_{0}^{X_{L}} k(\Delta v, X) N_{v}^{*}(X) \exp \left[-\int_{0}^{X} k\left(\Delta v, X^{\prime}\right) \mathrm{d} X^{\prime}\right] \mathrm{d} X \mathrm{~d}(\Delta v) \\
& W=\int_{-\infty}^{\infty}\left\{1-\exp \left[-\int_{0}^{X_{L}} k(\Delta v, X) \mathrm{d} X\right]\right\} \mathrm{d}(\Delta v) .
\end{aligned}
$$

The solutions require the separation of the path dependent and wavelength dependent terms in the absorption coefficient.

As in Ref. 1, two cases are now considered : (1) a "nearly weak" line approximation and (2) a "nearly strong" line approximation.

In the "thin gas" approximation (weak line case), equations (13) and (14) reduce to the simplified forms :

$$
N=\int_{0}^{x_{L}} N_{v}^{*}(X) S(X) \mathrm{d} X
$$

and

$$
W=\int_{0}^{x_{L}} S(X) d X
$$

Therefore, for weak lines, $N$ and $W$ are independent of $\gamma$. For "nearly weak" lines, the assumption that $N$ and $W$ are relatively insensitive to the value of $\gamma$ is made and we replace $\gamma(X)$ in equation (1) by an effective line half-width, $\gamma_{e}=$ constant, which may be taken to be a mass-weighted average line half-width, to obtain:

$$
k(v, X) \cong\left(\frac{\ln 2}{\pi}\right)^{1 / 2} \frac{S(X)}{\gamma_{e}} \exp \left[-\left(\frac{v-v_{0}}{\gamma_{e}}\right)^{2} \ln 2\right]
$$

The use of equation (17) is more reasonable than replacing the attenuation term in equation (13) by unity for nearly weak lines, where some self-absorption exists. It will later be shown that $N$ and $W$ for the nonisothermal case obtained by using this form of $k(v, X)$ reduces to the simple linear (weak line) forms, equations (6) and (7), for the isothermal condition and small optical depth. 
If equation (17) is a good approximation for nearly weak lines, we can certainly improve the approximation and extend its range of applicability by replacing $\gamma_{e}$ in the denominator by its actual value $\gamma(X)$ :

$$
k(v, X) \cong\left(\frac{\ln 2}{\pi}\right)^{1 / 2} \frac{S(X)}{\gamma(X)} \exp \left[-\left(\frac{v-v_{0}}{\gamma_{e}}\right)^{2} \ln 2\right] .
$$

In strong lines, further absorption takes place mostly in the line wings. For Doppler lines, the condition that $\left[\left(v-v_{0}\right) / \gamma\right]^{2} \gg 1$ means that the exponential in equation (1) cuts off absorption in the line wings very rapidly, no matter what value of $\gamma$ is used. Replacing $\gamma$ by $\gamma_{e}$ only in the exponential term to obtain equation (18) will therefore be termed the "nearly strong" line approximation. It will later be shown that $N$ and $W$ for the nonisothermal case obtained by using equation (18) reduces to the strong line forms, equations (9) and (10), for the isothermal condition and large optical depth.

The absorption coefficient can now be represented as:

$$
k(v, X)=\left(\frac{\ln 2}{\pi}\right)^{1 / 2} \frac{S(X) G(X)}{\gamma_{e}} \exp \left[-\left(\frac{v-v_{0}}{\gamma_{e}}\right)^{2} \ln 2\right]
$$

where $G(X)=1$ for the "nearly weak" line approximation and $G(X)=\left[\gamma_{e} / \gamma(X)\right]$ for the "nearly strong" line approximation.

Considering first the simpler expression $W$, we substitute equation (19) into equation (14):

$$
W=\int_{-\infty}^{\infty}\left[1-\exp \left\{-\int_{0}^{x_{\mathrm{L}}}\left(\frac{\ln 2}{\pi}\right)^{1 / 2} \frac{S(X) G(X)}{\gamma_{e}}\left(\exp \left[-\left(\frac{\Delta v}{\gamma_{e}}\right)^{2} \ln 2\right]\right) \mathrm{d} X\right\}\right] \mathrm{d}(\Delta v) .
$$

Define a dimensionless optical depth, $x$, by

$$
\begin{aligned}
\mathrm{d} x & =\left(\frac{\ln 2}{\pi}\right)^{1 / 2} \frac{S(X) G(X)}{\gamma_{e}} \mathrm{~d} X \\
x & =\left(\frac{\ln 2}{\pi}\right)^{1 / 2} \frac{1}{\gamma_{e}} \int_{0}^{x} S\left(X^{\prime}\right) G\left(X^{\prime}\right) \mathrm{d} X^{\prime}
\end{aligned}
$$

which upon substitution into equation (20) gives

$$
W=\int_{-\infty}^{\infty}\left[1-\exp \left\{-x_{L} \exp \left[-\left(\frac{\Delta v}{\gamma_{e}}\right)^{2} \ln 2\right]\right\}\right] \mathrm{d}(\Delta v)
$$

Expanding the first exponential on the right-hand side of equation (22) in an infinite series and interchanging the order of summation and integration, we obtain:

$$
\begin{aligned}
W & =\sum_{n=1}^{\infty} \frac{(-1)^{n-1}}{n !} x_{L}^{n} 2 \int_{0}^{\infty}\left\{\exp \left[-n\left(\frac{\Delta v}{\gamma_{e}}\right)^{2} \ln 2\right]\right\} \mathrm{d}(\Delta v) \\
& =\left(\frac{\pi}{\ln 2}\right)^{1 / 2} \gamma_{e} \sum_{n=0}^{\infty} \frac{(-1)^{n} x_{L}^{n+1}}{(n+1) !(n+1)^{1 / 2}} .
\end{aligned}
$$


Identifying the infinite series on the right-hand side of equation (23) as the function $g$ defined in equation (4), which was obtained in the solution for $W$ and $N$ for the isothermal case, we obtain the desired expression for the equivalent width of a nonisothermal Doppler line:

$$
W=\left(\frac{\pi}{\ln 2}\right)^{1 / 2} \gamma_{e} g\left(x_{L}\right)
$$

Substituting equations (19) and (21) into equation (13):

$$
N=\int_{-\infty}^{\infty} \int_{0}^{x_{L}} N_{v}^{*}(x)\left[\exp \left\{-\left(\frac{\Delta v}{\gamma_{e}}\right)^{2} \ln 2\right\}\right] \exp \left\{-x \exp \left[-\left(\frac{\Delta v}{\gamma_{e}}\right)^{2} \ln 2\right]\right\} \mathrm{d}(\Delta v) \mathrm{d} x
$$

Expanding the second exponential on the right-hand side of equation (25) in an infinite series and interchanging the order of summation and integration, we obtain :

$$
\begin{aligned}
N & =\int_{0}^{x_{L}} N_{v}^{*}(x) 2 \sum_{n=0}^{\infty} \frac{(-1)^{n}}{n !} x^{n} \int_{0}^{\infty}\left\{\exp \left[-(n+1)\left(\frac{\Delta v}{\gamma_{e}}\right)^{2} \ln 2\right]\right\} \mathrm{d}(\Delta v) \mathrm{d} x \\
& =\left(\frac{\pi}{\ln 2}\right)^{1 / 2} \gamma_{c} \int_{0}^{x_{L}} N_{v}^{*}(x) \sum_{n=0}^{\infty} \frac{(-1)^{n}}{n !} \frac{x^{n}}{(n+1)^{1 / 2}} \mathrm{~d} x .
\end{aligned}
$$

We recognize the infinite series in equation (26) to be the derivative of $g$ :

$$
\frac{\mathrm{d} g}{\mathrm{~d} x}=\sum_{n=0}^{\infty} \frac{(-1)^{n} x^{n}}{n !(n+1)^{1 / 2}}
$$

Substituting equation (27) into equation (26), we obtain the desired expression for the total radiance of a nonisothermal Doppler line:

$$
N=\left(\frac{\pi}{\ln 2}\right)^{1 / 2} \gamma_{e} \int_{0}^{g\left(x_{L}\right)} N_{v}^{*}(g) \mathrm{dg}
$$

The function $g(x)$ has asymptotic values of $x$ and $(2 / \sqrt{ } \pi)[\ln (x)]^{1 / 2}$ for small $x$ and large $x$, respectively. The corresponding representations for $\mathrm{d} g$ are $\mathrm{d} x$ and $x^{-1}[\pi \ln (x)]^{-1 / 2} \mathrm{~d} x$. Substituting $g=x$ and $\mathrm{d} g=\mathrm{d} x$ into cquations (24) and (28), we obtain the nonisothermal weak line expressions:

$$
\begin{gathered}
W=\left(\frac{\pi}{\ln 2}\right)^{1 / 2} \gamma_{e} x_{L}=\int_{0}^{x_{L}} S(X) \mathrm{d} X, \\
N=\left(\frac{\pi}{\ln 2}\right)^{1 / 2} \gamma_{e} \int_{0}^{x_{L}} N_{v}^{*}(x) \mathrm{d} x=\int_{0}^{x_{L}} N_{v}^{*}(X) S(X) \mathrm{d} X .
\end{gathered}
$$


Under isothermal conditions, equations (29) and (30) reduce to equations (6) and (7), the weak line isothermal equations for $N$ and $W$ for optical depth $X=X_{L}$. Substituting $g=(2 / \sqrt{ } \pi)[\ln (x)]^{1 / 2}$ and $\mathrm{d} g=x^{-1}[\pi \ln (x)]^{-1 / 2} \mathrm{~d} x$ into equations (24) and (28), we obtain the nonisothermal strong line expressions:

$$
W=\left(\frac{\pi}{\ln 2}\right)^{1 / 2} \gamma_{e} \frac{2}{\sqrt{\pi}}\left[\ln \left(x_{L}\right)\right]^{1 / 2}=\frac{2 \gamma_{e}}{(\ln 2)^{1 / 2}}\left\{\ln \left[\left(\frac{\ln 2}{\pi}\right)^{1 / 2} \frac{1}{\gamma_{e}} \int_{0}^{x_{L}} S(X) G(X) \mathrm{d} X\right]\right\}^{1 / 2}
$$

and

$$
\begin{aligned}
N= & \left(\frac{\pi}{\ln 2}\right)^{1 / 2} \gamma_{e} \int_{0}^{x_{L}} N_{v}^{*}(x) x^{-1}[\pi \ln (x)]^{-1 / 2} \mathrm{~d} x \\
= & \frac{\gamma_{e}}{(\ln 2)^{1 / 2}} \int_{0}^{x_{L}} N_{v}^{*}(X)\left[\int_{0}^{x} S\left(X^{\prime}\right) G\left(X^{\prime}\right) \mathrm{d} X^{\prime}\right]^{-1} \\
& \times\left[\ln \left\{\left(\frac{\ln 2}{\pi}\right)^{1 / 2} \frac{1}{\gamma_{e}} \int_{0}^{X} S\left(X^{\prime}\right) G\left(X^{\prime}\right) \mathrm{d} X^{\prime}\right\}\right]^{-1 / 2} S(X) G(X) \mathrm{d} X .
\end{aligned}
$$

\section{TABLE 1. SUMmaRY OF RELATIONS}

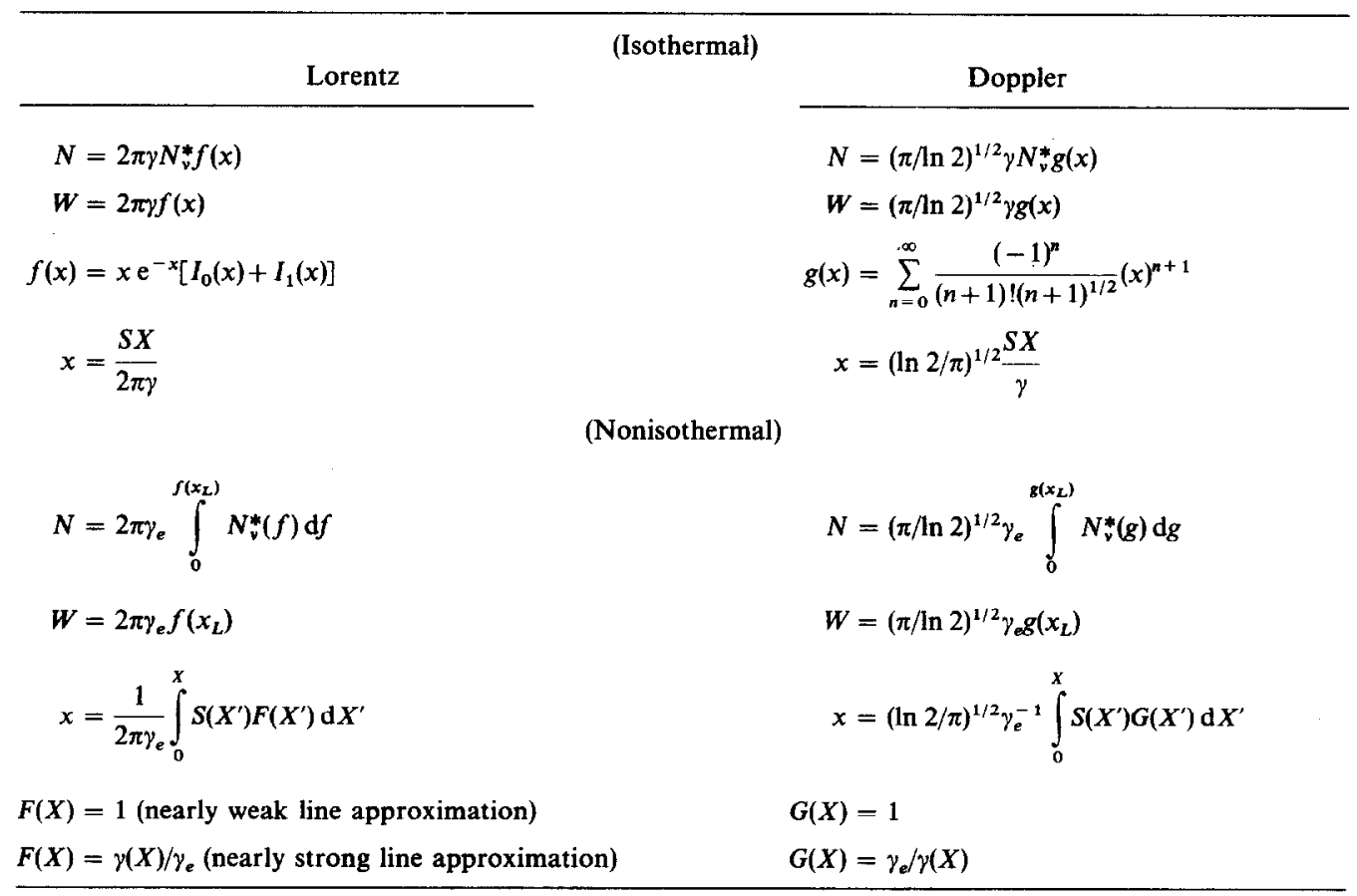


Under isothermal conditions, equations (31) and (32) reduce to the following forms:

$$
\begin{gathered}
W=\frac{2 \gamma}{(\ln 2)^{1 / 2}}\left\{\ln \left[\left(\frac{\ln 2}{\pi}\right)^{1 / 2} \frac{S X_{L}}{\gamma}\right]\right\}^{1 / 2} \\
N=\frac{\gamma}{(\ln 2)^{1 / 2}} N_{v}^{*} \int_{0}^{x_{L}} X^{-1}\left[\ln \left\{\left(\frac{\ln 2}{\pi}\right)^{1 / 2} \frac{S X}{\gamma}\right\}\right]^{-1 / 2} \mathrm{~d} X \\
=\frac{2 \gamma}{(\ln 2)^{1 / 2}} N_{v}^{*}\left[\ln \left\{\left(\frac{\ln 2}{\pi}\right)^{1 / 2} \frac{S X_{L}}{\gamma}\right\}\right]^{1 / 2}
\end{gathered}
$$

which are identical to equations (9) and (10), the strong line isothermal equations for $N$ and $W$, for optical depth $X=X_{L}$.

It is interesting to note the similarities between the isothermal and nonisothermal relations for $N$ and $W$ for the Lorentz and Doppler lines, as summarized in Table 1.

\section{REFERENCES}

1. F. S. SMMONS, JQSRT 7, 111 (1967).

2. S. S. PenNer, Quantitative Molecular Spectroscopy and Gas Emissivities. Addison-Wesley, Mass. (1959).

3. G. H. Hardy, London Math. Soc. Proc. 2, 401 (1905).

4. R. Ladenburg, Z. Phys. 65, 200 (1930). 